\section{$\underset{\substack{\text { hommes } \\ \text { \& migrations }}}{ }$}

\section{Hommes \& migrations}

Revue française de référence sur les dynamiques

migratoires

$1283 \mid 2010$

Cuisines et dépendances

\title{
La Cocotte-minute entre la France et le Maghreb
}

Regards de femmes sur un objet culinaire

\section{Aurélie Brayet}

\section{(e) OpenEdition}

12 Journals

\section{Édition électronique}

URL : http://journals.openedition.org/hommesmigrations/1000

DOI : 10.4000/hommesmigrations. 1000

ISSN : 2262-3353

Éditeur

Musée national de l'histoire de l'immigration

\section{Édition imprimée}

Date de publication : 1 janvier 2010

Pagination : 128-134

ISBN : 978-2-919040-04-9

ISSN : $1142-852 X$

\section{Référence électronique}

Aurélie Brayet, «La Cocotte-minute entre la France et le Maghreb», Hommes \& migrations [En ligne], 1283 | 2010, mis en ligne le 29 mai 2013, consulté le 19 avril 2019. URL : http://

journals.openedition.org/hommesmigrations/1000; DOI : 10.4000/hommesmigrations.1000 


\section{La Cocotte-minute entre la France et le Maghreb}

\section{Regards de femmes sur un objet culinaire}

Par Aurélie Brayet, IERP, université Jean Monnet, Saint-Etienne

En France, la majorité des ménages possèdent un autocuiseur, plus couramment appelé "Cocotte-minute". Cet objet, considéré comme l'expression du "chic français" dans certains pays comme

le Japon ou la Chine, rencontre un succès sans précédent au Maghreb. Depuis une dizaine d'années, son fabriquant y réalise la plupart de ses ventes. La Cocotte, particulièrement appropriée à la cuisine maghrébine, est entrée dans les mœurs. Sa simplicité d'utilisation, son caractère intuitif et la possibilité de mijoter longuement les plats en font l'ustensile vedette de la ménagère. 
À l'heure où l'on souhaite classer la gastronomie française au patrimoine mondial de l'UNESCO, une question se pose sur le caractère "français" d'une technique de cuisson exportée dans une ancienne colonie et en terre d'immigration. On dit souvent que l'un des plats préférés des Français, le couscous, vient de l'autre rive de la Méditerranée. La présente étude propose de se placer du point de vue des cuisinières maghrébines, d'aller à la rencontre de ces femmes (et parfois de ces hommes) qui, chaque jour, préparent des repas familiaux en France. L'enquête se fonde sur un objet mythique de l'industrie et des cuisines françaises, présenté notamment en Chine en 2004, lors de l'année de la France en Chine, comme le produit français par excellence : l'autocuiseur.

Nous avons rencontré une trentaine de familles françaises immigrées et originaires du Maghreb (plus particulièrement de Turquie, d'Algérie et du Maroc) utilisant l'autocuiseur. Quelles sont les raisons d'un tel achat? Quelles sont les utilisations de cet ustensile ? Que cuisine-t-on avec ? Des plats français "à la française" ? Des plats français "à l'algérienne" ? Des plats du pays "à l'algérienne" ? Nous avons volontiers laissé la parole aux utilisatrices et utilisateurs de l'autocuiseur. Il s'agissait de s'immerger dans les cuisines de nos témoins, femmes et hommes, liés par le cceur, le sang et la cuisine à l'Algérie, la Turquie ou le Maroc. Et de se confronter avant tout à la pratique de la cuisson. L'enquête s'est déroulée durant un mois, à Saint-Etienne ${ }^{(1)}$. Les Stéphanois interrogés sont donc au centre de notre étude sur l'autocuiseur. Il s'agit ici de comprendre les usages de l'autocuiseur, mais aussi d'en déchiffrer les significations et les processus d'identification.

\section{Familles stéphanoises et Cocotte-minute : mise en place de l'enquête}

La rencontre avec les cuisines maghrébines est riche d'enseignements sur les rapports aux innovations et l'inscription dans un discours explicatif, voire idéologique. Pour étudier les usages de l'autocuiseur en France par les communautés algériennes, turques et marocaines, il a fallu aller à la rencontre des usagers. Plusieurs possibilités de mise en contact sont alors possibles. Le territoire stéphanois semblait offrir un cadre idéal à cette enquête, notamment parce que l'agglomération stéphanoise regroupe de nombreux ressortissants maghrébins et abrite sur son sol un consulat d'Algérie.

La création d'un blog ${ }^{(2)}$ et la diffusion d'un appel à témoin ont permis de recueillir 54 témoignages écrits utilisables. Ils ont permis d'affiner le questionnaire en vue de la rencontre des membres du Club féminin de Montchovet à Saint-Etienne, une 
association qui a pour objectif l'alphabétisation des femmes du Maghreb arrivées à Saint-Etienne plus ou moins récemment. L'équipe est composée exclusivement de femmes (mais fait appel parfois, pour certaines activités, à des bénévoles hommes), ce qui donne lieu à une certaine convivialité et complicité. Situé au cceur du quartier de Montchovet, le Club est un lieu d'échange et d'accueil où rien n'est imposé : toutes les femmes du quartier passent volontiers dans les locaux avant ou après les courses, ou pour des activités particulières. C'est lors de ces moments d'échange que l'équipe aborde les questions d'alphabétisation.

L'équipe féminine a travaillé avec plaisir sur le sujet de l'autocuiseur, bon moyen d'échange et de discussion. Marie, Nathalie, Corinne, Norah et Leïla ont aidé à nouer le contact, en vue d'organiser une journée d'échange sur le sujet de l'autocuiseur le 24 septembre 2009. Pour laisser la parole à chacune des femmes rencontrées collectivement puis individuellement, voici la retranscription de leurs propos.

\section{Fatima : "La Cocotte-minute, je vois pas comment on peut cuisiner sans."}

Née à Alger en 1955, Fatima fête cette année ses trente-trois ans de mariage. Mère au foyer, elle partage la vie de Rachid, son mari, qui est électricien et maçon. Elle est la mère de sept enfants, trois garçons et quatre filles. L'entretien individuel a eu lieu chez elle à Saint-Etienne, quartier Montchovet, dans le même immeuble que le Club. "En ce qui concerne la cuisine, la Cocotte-minute, c'est sacré ! Petite, j’en ai toujours vu au moins une dans la cuisine de ma mère. Mes sceurs étaient plus grandes, c'est plutôt elles qui s'en servaient, avec ma mère. Moi, maintenant, j'en ai deux, une petite et une grande. [...] La cuisine, c'est quelque chose d'important, c'est vraiment quelque chose qu'il faut bien faire."

Côté qualité des appareils, elle préfère les Cocottes vendues en Algérie. "En France, les SEB, sont très chères et elles font du bruit. Il y a de l'électronique dedans, c'est pour ça. Ma fille aînée en a une comme ça. Elle fait un bruit pas possible et il n'y a plus de soupape qui tourne et qui fait 'tchoutchou."'

Elle demeure très traditionnelle dans sa cuisine. "J'ai un micro-ondes, mais moi je ne m'en sers que pour chauffer l'eau. Mon mari se fait parfois des portions avec. Il prend un reste et il le met au micro-ondes. Mais pas moi. Je préfêre manger quand ça sort de la Cocotte. J'ai toujours cuisiné avec. Au moins la viande est bien cuite et il faut que la viande soit bien cuite". Chez Fatima, pas de livre de recettes ni de minutage des temps de cuisson. "Il y a très peu de temps que jarrive à lire et puis, dans la cuisine algérienne, tout est affaire de jugé. Moi, je fais mon ménage et là je sais que c'est cuit. J'ai toujours cuisiné comme ça. La cuisine, il faut la sentir. Quand tu fais une viande, il faut la laisser très longtemps. Il faut bien que ça sente la viande. Pour les légumes, c'est pareil, mais c'est moins long. Il faut juste faire 
attention à mettre toujours de l'eau, sinon ça peut coller et après ça brûle. Pas besoin de regarder l'heure, de toute façon si ce n'est pas assez cuit, tu remets après avec les légumes et le jus."

\section{Zakia : "Ça permet de tout cuire ensemble et de vraiment bien cuire."}

Zakia est née à Bejaya en Algérie, en mars 1964. Aînée d'une famille de six enfants, elle est arrivée en France il y a treize ans pour son mariage. Son mari est chauffeur de poids lourds. De vingt ans son aîné, il est souvent absent pour son travail et Zakia tient à s'occuper avec sérieux de ses trois enfants. Elle a toujours vécu à la ville dans un grand appartement. Elle aime la cuisine, le crochet et la télévision.

"Je cuisine surtout avec la Cocotte. D’ailleurs, je cuis tout, la viande, le couscous, les légumes. Ma voisine, une Chinoise, m'a donné un panier en fer avec des trous pour mettre sur la Cocotte. Du coup, je fais ma semoule comme ça pour les jours en semaine. Je mets de l'eau au fond, le panier et la semoule. Sinon, je fais des viandes, avec des pommes de terre et des tomates. La Cocotte, c'est bien, ça permet de tout cuire ensemble et de vraiment bien cuire." Comme Fatima, elle fonctionne au jugé et se passe volontiers de livre de recettes. "Moi, la cuisine, c'est comme ça, je fais ce que je sais faire. Limportant, c'est de mettre les choses dans l'ordre, et de cuire longtemps. Et puis de bien mettre les épices. Au début, jai dû refaire cuire des viandes. Mais depuis, je cuisine comme avant de l'avoir. Je fais les recettes que ma mère m'a apprises, mais dans la Cocotte, c'est tout. Ça cuit mieux et c'est bien."

\section{Mina : "C"est un objet algérien avant tout. Nous nous le sommes appropriés."}

Mina est née à Maison-Lafitte en 1977. Elle est assistante de projet à Saint-Etienne et son mari est agriculteur dans le Pilat. Elle a rencontré son mari dans sa famille stéphanoise. Mère de deux enfants, elle est fière de ses origines algériennes. Ses parents sont nés à Baghlia (campagne). Ils étaient ouvriers agricoles. Seule fille élevée avec ses trois frères, elle est partie du domicile parental en 2004 après des études de droit. Elle dit aimer la cuisine et y consacrer quarante minutes pour chaque repas. "Pour moi, la cuisine c'est important. Mon mari aime les plats bien cuisinés. Du coup, quand je me suis mariée jai repris les recettes de ma mère. Ça ne s'oublie pas la cuisine de l'enfance. Et puis avec trois frères à la maison, on cuisinait beaucoup avec ma mère. [...] La Cocotte, ma mère en avait plusieurs, alors pour moi c'était naturel. Quand je suis partie, ma mère m'a donné une des siennes, que j’ai toujours. Et puis j’en ai eu une autre à mon mariage." Elle cuisine des recettes de famille, des ragoûts surtout. "Ce sont des plats typiques d'Algérie que ma mère faisait et que je fais. Sinon, non, je ne regarde pas les livres de recettes. 
J'en ai quelques-uns de cuisine 'française', mais c'est plus pour des idées. Ma spécialité, c'est la jardinière de légumes au lapin. C'est une recette française, avec du porc normalement, et moi je la fais avec du lapin. C'est très bon. [...] Pour moi, la Cocotte-minute c'est un gain de temps considérable. Tout y cuit plus vite. Et puis, c'est toujours mieux cuit."

Pour elle qui connaît la France et l'Algérie, ses "deux pays", la cocotte-minute n'a rien de proprement français. "Quand je vivais à Maison-Lafitte, je n'ai jamais vu les mamans de mes amies utiliser une Cocotte-minute. Par contre, c'est un objet courant en Algérie. Le bruit du souffle qui s'échappe de la Cocotte lors de la cuisson est un bruit très familier en Algérie. Je dirais que c'est un objet algérien avant tout. Nous nous le sommes appropriés. Non, cet objet n'a aucune connotation française pour moi, vraiment pas. D'ailleurs, jai cru jusqu'à très récemment que c'était une invention de chez nous, qui s'était implantée en France dans les familles algériennes."

\section{Rachid : "Je trouve cette Cocotte, comment dire... rassurante. Pour moi, la cuisine, c'est la Cocotte-minute..."}

Rachid est né à Saint-Etienne, ses parents à Tanger. Il est guide conférencier dans un musée stéphanois. Âgé de 30 ans, il est parti de chez ses parents et vit depuis peu en couple avec Mathilde.

"Quand je suis parti de la maison, il y a trois ans, ma mère m'a entièrement équipé la cuisine. Ce qui est drôle c'est que j’ai un frigo-congélateur, des passoires, un couscoussier, des tonnes de petits robots, un micro-ondes, mais qu'elle n'a pas mis de Cocotte-minute dans ma cuisine! Quand je lui ai demandé des astuces la première fois que j’ai voulu faire une viande en sauce, surtout sur les temps de cuisson... Elle m'a tout expliqué, que, elle, elle le fait dans sa cocotte! Et moi je n'avais pas d'autocuiseur! Je me suis un peu rebellé parce qu'elle en avait offert un à ma sæur pour son installation et que javais beau avoir un tas de trucs partout dans les placards, là, il n'y avait pas l'essentiel, cet autocuiseur magique qui fait les viandes super bonnes de ma mère! Je trouve cette Cocotte, comment dire..., rassurante. Pour moi, la cuisine de maman, c'est la Cocotte. Du coup, pendant deux ans, je n'ai mangé en semaine que des plats vite préparés, type pâtes, viande, poisson, légumes... Sans Cocotte, je ne pouvais pas faire les plats marocains de maman."

Pour autant, Rachid n'a pas acheté lui-même de Cocotte. "C'est comme si c'était un truc de femme en fait. Il y a des espèces de secrets pour l'utiliser. Quand jétais à la maison, ma mère ne m'a jamais montré comment ça marche!' C'est sa compagne, Mathilde, venue s'installer dans son appartement il y a un an, qui lui en a expliqué le fonctionnement. "Elle est arrivée avec sa Cocotte-minute, un cadeau de sa mère! Elle cuisine tout le temps avec, et je m'y suis mis. Je fais des plats typiques, comme elle dit. Je fais des tajines et des couscous quand on reçoit des amis ou la famille. Mathilde m'a expliqué 
pour les temps de cuisson. Ils sont indiqués pour chaque aliment à la fin du mode d'emploi. C'est très pratique. J'ai même l'impression de cuisiner plus vite que ma mère certains plats, mais je crois qu'elle ne regarde pas le livre, elle, et puis elle n'a pas le même modèle, c'est peut-être pour ça..."

\section{Assia, Fatima et Djamila : "C'est bon, c'est ça qui est important."}

Assia est née à Constantine. Elle est arrivée à 17 ans en France pour le mariage. Mère de quatre enfants, elle est âgée de 53 ans. Elle revient sur l'omniprésence de la Cocotte en Algérie. "En Algérie, la Cocotte, c'est partout. À Constantine, moi jen ai toujours vu. Il y a même une chanson dessus. C'est une discussion entre la Cocotte et une marmite. Ça dit que la cuisson est trop longue et qu'avec la Cocotte c'est parfait. C'est un écrivain qui a écrit cette histoire."

Djamila est née à Alger. Âgée de 48 ans, elle est arrivée en France avec son mari à 29 ans. Elle est mère de trois enfants. Avec elle, comme chez les autres femmes interviewées, la Cocotte se passe de livre de recettes. Son fonctionnement a fait l'objet d'une transmission familiale. "La cuisine, c'est au jugé. Pourquoi tu veux que l'on t'explique? Une Cocotte, il faut mettre de l'eau et bien la fermer. Après, c'est parti. Moi, je fais la cuisine de ma famille avec les recettes de famille et c'est bon, c'est ça qui est important." Pour Fatima - âgée de 49 ans et mère de cinq enfants, née à Alger et arrivée en France à 20 ans pour le mariage -, la Cocotte permet de satisfaire à un impératif religieux en matière de cuisine : éliminer plus radicalement le sang restant dans la viande. "Chez nous, il ne faut pas de sang dans notre cuisine. Pour le poulet, je le fais cuire 30 minutes à la Cocotte, puis je mets au four pendant 15 à 20 minutes. Il ne faut pas mettre juste comme ça au four, sinon ce n'est pas assez cuit. Avec la Cocotte, c'est parfait comme cuisson."

\section{Conclusion}

Au travers de ces quelques retranscriptions de témoignages tirés d'un corpus plus large, on se rend compte des spécificités de l'usage de l'autocuiseur dans les communautés maghrébines. Au-delà d'un usage répété, courant, et d'un attachement à l'objet que l'on retrouve chez les utilisatrices de culture culinaire française, ainsi qu'un engouement pour l'économie de temps et d'argent que permet l'ustensile, la Cocotte-minute est un élément essentiel, fondamental des cultures culinaires du Maghreb. La Cocotte est rassurante. C'est un partenaire intime qui s'est laissé apprivoiser malgré les mauvaises expériences. La Cocotte-minute est utilisée et vantée pour la perfection de sa cuisson 
des plats typiques. On parle souvent de cuisson "parfaite". La cocotte est donc un objet parfaitement adapté à la cuisine du Maghreb et à la vie moderne. Elle permet, en effet, de faire plus vite et sans surveillance une cuisine qui doit être longtemps mijotée. À l'inverse des utilisatrices interviewées en 2004 pour l'écriture de l'histoire de l'autocuiseur, chez nos témoins du Club féminin, on remarque, d'une part, que l'usage de l'autocuiseur s'apprend par transmission mère/fille, ou de la sceur à la belle-sceur. C'est un peu comme si la fonction d'épouse imposait l'apprentissage culinaire, une sorte de passage au statut de femme accompagné par la Cocotte. D'autre part, l'objet est perçu comme étant "au service de" et non comme demandant une formation technique. Ainsi, l'usage du livre de cuisine, et surtout le respect des temps de cuissons proposés par le manuel, sont totalement absents chez nos témoins. De même, on ouvre et ferme à sa guise l'autocuiseur, on ne se plie pas à la technique mais on l'adapte à sa cuisine. Enfin, s'il ressort de cette enquête un sentiment d'affection envers la Cocotte-minute, on découvre aussi qu'elle est un élément de l'identité culinaire du Maghreb.

- "Manger au Maghreb", Horizons maghrébins. Le droit à la mémoire, n55, Presses universitaires du Mirail, 2006.

- Hélène d'Almeida-Topor, Le goût de l'étranger. Les saveurs venues d'ailleurs depuis la fin du XVIII' siècle, Paris, Armand Colin, 2006.

- Aurélie Brayet, Ma Cocotte bien-aimée. Histoire et mémoire d'un objet quotidien, Saint-Etienne, PUSE, 2009.

- Michel de Certeau, Linvention du quotidien : habiter, cuisiner, tome 2, Paris, Folio, 1999.

- Jean-Claude Kaufmann, L'entretien compréhensif, Paris, Armand Colin, 2004.

- Jean-Claude Kaufmann, La vie ordinaire, voyage au coeur du quotidien, Paris, Éditions Greco, 1989.

- Mille et une bouches, cuisines et identités culturelles, Paris, Autrement, 1995.

- Yvonne Verdier, "Pour une ethnologie culinaire", L'Homme, janvier-mars 1969.

\section{Notes}

1. Nous proposons ici la partie "française" d'une enquête réalisée avec Nabila Mokrani. Liées d'amitié lors d'une rencontre dans un colloque à Aix-en-Provence, nous avions projeté d'étudier et d'échanger sur le thème de l'autocuiseur en confrontant nos regards sur deux territoires, l'Algérie et la France. Notre travail a donné lieu à un article en cours de parution dans la revue Anthropoly of Food. Avec son accord, voici le résultat de l'enquête élargie aux ressortissants du Maghreb, que j'ai réalisé en France. Je tiens à remercier ici Nabila pour cet échange passionnant sur la cuisine et le plaisir que j'ai eu à partager et confronter nos deux études transméditerranéennes.

2. (http://cocotte-minute.cuisineblog.fr) 